\title{
DeeplyTough: Learning Structural Comparison of Protein Binding Sites (Supporting Information)
}

\author{
Martin Simonovsky*,+,, and Joshua Meyers*,† \\ $\dagger$ BenevolentAI, London W1T 5HD, United Kingdom \\ $\ddagger E$ Eole des Ponts ParisTech, 77455 Champs sur Marne, France \\ ๑Université Paris-Est, 77455 Champs sur Marne, France \\ E-mail: martin.simonovsky@enpc.fr; joshua.meyers@benevolent.ai
}



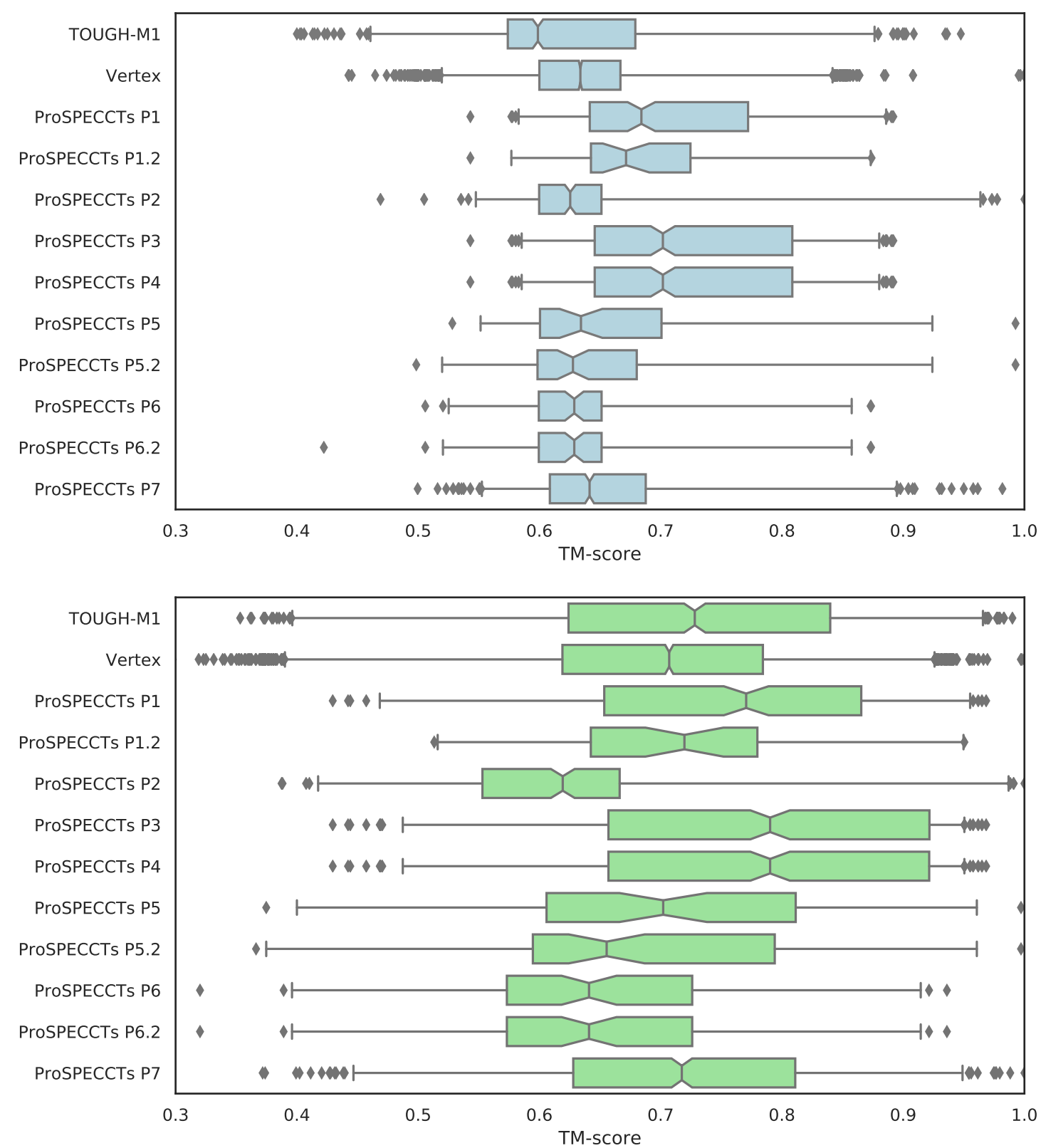

Figure S1: Box plots of TM-scores indicating the structurally similarity of pockets in a test set to their nearest neighbor pockets in a training set (again, in terms of TM-score) for each training scenario in this work. For TOUGH-M1 dataset, a single random permutation out of ten is visualized. The scores are computed with TM-align ${ }^{1}$, with 1.0 indicating identity and 0.0 no alignment. Whiskers mark $1 \%$ and $99 \%$ percentiles of a distribution. Pockets in TOUGH-M1 can be defined in two ways: i) computationally with Fpocket $2.0^{2}$ as used for training DeeplyTough (blue plots; top), or ii) around their bound ligands as in Vertex and ProSPECCTs (green plots; bottom). In general, the distributions suggest that there is no strong contamination between training and test sets (especially with computationally defined pockets), with only several pocket pairs being near-identical. 


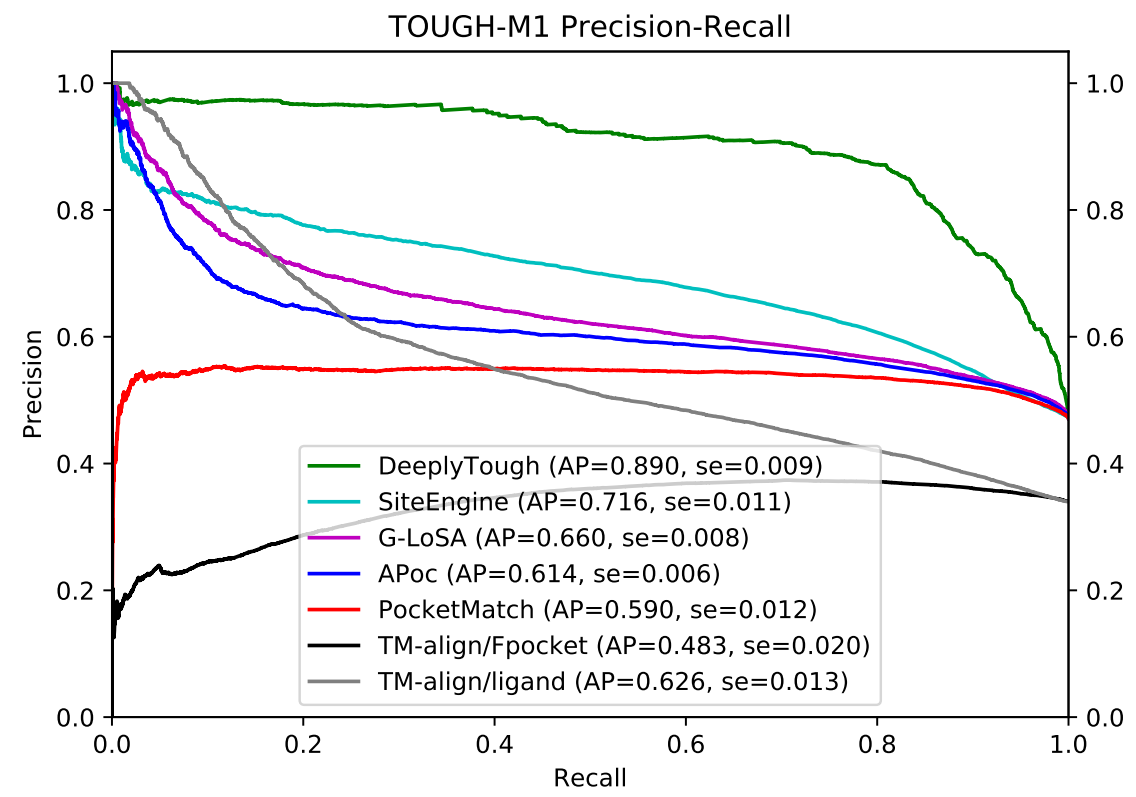

Figure S2: Precision-recall plot with associated average precision (AP) values evaluating the performance of pocket matching algorithms on TOUGH-M1 testing folds. Standard error, denoted as se, is measured over ten random splits.

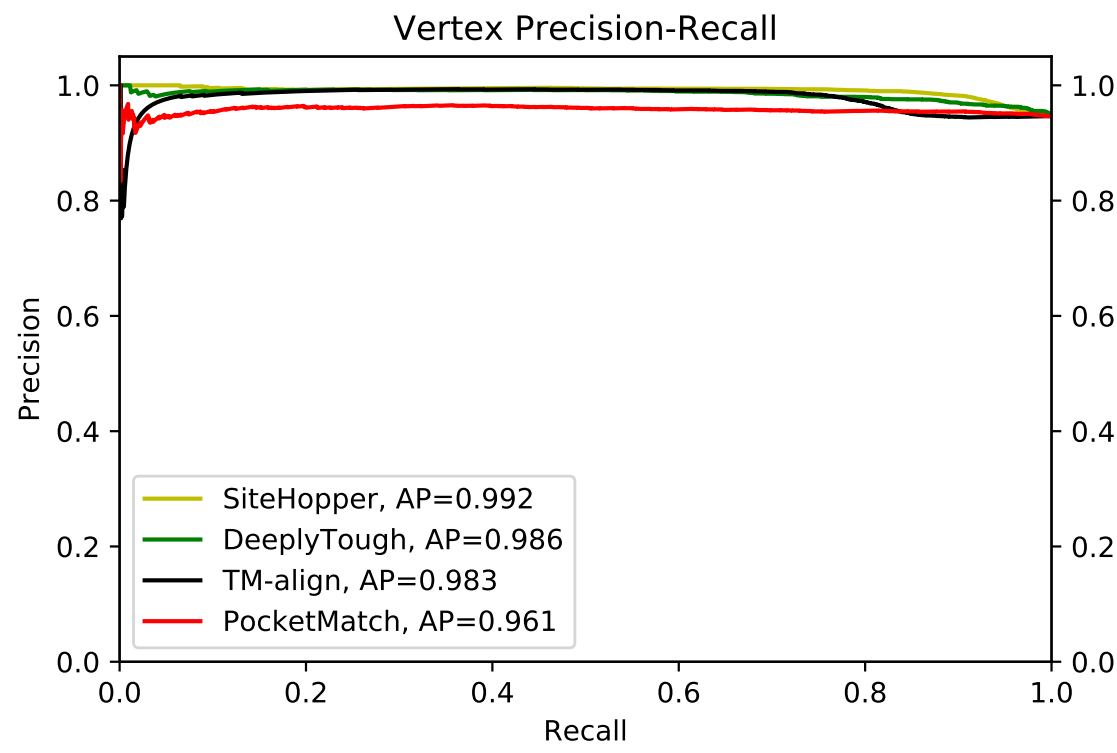

Figure S3: Precision-recall plot with associated average precision (AP) values evaluating the performance of pocket matching algorithms on the Vertex dataset (6,977 protein pairs). 
Table S1: AP values for DeeplyTough on each of ten ProSPECCTs datasets.

\begin{tabular}{c|cccccccccc} 
& $\mathrm{P} 1$ & $\mathrm{P} 1.2$ & $\mathrm{P} 2$ & $\mathrm{P} 3$ & $\mathrm{P} 4$ & $\mathrm{P} 5$ & $\mathrm{P} 5.2$ & $\mathrm{P} 6$ & $\mathrm{P} 6.2$ & P7 \\
\hline DeeplyTough & 0.78 & 0.90 & 0.57 & 0.76 & 0.77 & 0.33 & 0.28 & 0.37 & 0.37 & 0.45
\end{tabular}

\section{References}

(1) Zhang, Y.; Skolnick, J. TM-align: A Protein Structure Alignment Algorithm Based on the TM-score. Nucleic acids research 2005, 33, 2302-2309.

(2) Le Guilloux, V.; Schmidtke, P.; Tuffery, P. Fpocket: An Open Source Platform for Ligand Pocket Detection. BMC Bioinform. 2009, 10. 